\title{
ON HIGHER HEINE-STIELTJES POLYNOMIALS
}

\author{
THOMAS HOLST AND BORIS SHAPIRO
}

\begin{abstract}
Take a linear ordinary differential operator $\mathfrak{d}(z)=\sum_{i=1}^{k} Q_{i}(z) \frac{d^{i}}{d z^{i}}$ with polynomial coefficients and set $r=\max _{i=1, \ldots, k}\left(\operatorname{deg} Q_{i}(z)-i\right)$. If $\mathfrak{d}(z)$ satisfies the conditions: i) $r \geq 0$ and ii) $\operatorname{deg} Q_{k}(z)=k+r$ we call it a nondegenerate higher Lamé operator. Following the classical examples of E. Heine and T. Stieltjes we initiated in [6] the study of the following multiparameter spectral problem: for each positive integer $n$ find polynomials $V(z)$ of degree at most $r$ such that the equation:

$$
\mathfrak{d}(z) S(z)+V(z) S(z)=0
$$

has a polynomial solution $S(z)$ of degree $n$. We have shown that under some mild non-degeneracy assumptions on $T$ there exist exactly $\left(\begin{array}{c}n+r \\ n\end{array}\right)$ spectral polynomials $V_{n, i}(z)$ of degree $r$ and their corresponding eigenpolynomials $S_{n, i}(z)$ of degree $n$. Localization results of [6] provide the existence of abundance of converging as $n \rightarrow \infty$ sequences of normalized spectral polynomials $\left\{\widetilde{V}_{n, i_{n}}(z)\right\}$ where $\widetilde{V}_{n, i_{n}}(z)$ is the monic polynomial proportional to $V_{n, i_{n}}(z)$. Below we calculate for any such converging sequence $\left\{\widetilde{V}_{n, i_{n}}(z)\right\}$ the asymptotic rootcounting measure of the corresponding family $\left\{S_{n, i_{n}}(z)\right\}$ of eigenpolynomials. We also conjecture that the sequence of sets of all normalized spectral polynomials $\left\{\widetilde{V}_{n, i}(z)\right\}$ having eigenpolynomials $S(z)$ of degree $n$ converges as $n \rightarrow \infty$ to the standard measure in the space of monic polynomials of degree $r$ which depends only on the leading coefficient $Q_{k}(z)$.
\end{abstract}

\section{INTRODUCTION}

A generalized Lamé equation, see e.g. [20] is the second order differential equation given by:

$$
Q_{2}(z) \frac{d^{2} S}{d z^{2}}+Q_{1}(z) \frac{d S}{d z}+V(z) S=0
$$

where $Q_{2}(z)$ is a complex polynomial of degree $l$ and $Q_{1}(z)$ is a complex polynom of degree at most $l-1$. If we fix the polynomials $Q_{2}(z)$ and $Q_{1}(z)$ then the classical Heine-Stieltjes spectral problem, [8], [15] asks to determine for any given positive integer $n$ all possible polynomials $V(z)$ such that (1.1) has a polynomial solution $S(z)$ of degree $n$. Such $V(z)$ are referred to as Van Vleck polynomials and their corresponding polynomials $S(z)$ are called Stieltjes or Heine-Stieltjes polynomials.

The next fundamental proposition announced in [8] was the starting point of the Heine-Stieltjes theory. (Notice that throughout this paper we count polynomials $V(z)$ individually and polynomials $S(z)$ projectively, i.e. up to a non-vanishing constant factor.)

Theorem 1 (Heine). If the polynomials $Q_{2}(z)$ and $Q_{1}(z)$ are algebraically independent, i.e. their coefficients do not satisfy an algebraic equation with integer coefficients then for any integer $n>0$ there exists exactly $\left(\begin{array}{c}n+l-2 \\ n\end{array}\right)$ polynomials

Date: April 1, 2009.

1991 Mathematics Subject Classification. 30C15; 31A15, 34E99.

Key words and phrases. Lamé equation, Van Vleck and Heine-Stieltjes polynomials, asymptotic root-counting measure. 
$V(z)$ of degree exactly $(l-2)$ such that the equation (1.1) has and unique (up to a non-vanishing constant factor) polynomial solution $S(z)$ of degree exactly $n$.

In [6] we generalize problem (1.1) to high order operators as follows. Consider an arbitrary linear ordinary differential operator

$$
\mathfrak{d}(z)=\sum_{i=1}^{k} Q_{i}(z) \frac{d^{i}}{d z^{i}},
$$

with polynomial coefficients. The number $r=\max _{i=1, \ldots, k}\left(\operatorname{deg} Q_{i}(z)-i\right)$ is called the Fuchs index of $\mathfrak{d}(z)$. The operator $\mathfrak{d}(z)$ is called a higher Lamé operator if its Fuchs index $r$ is nonnegative. In the case of the vanishing Fuchs index $\mathfrak{d}(z)$ is usually called exactly solvable in the physics literature. The operator $\mathfrak{d}(z)$ is called non-degenerate if $\operatorname{deg} Q_{k}(z)=k+r$.

Given a higher Lamé operator $\mathfrak{d}(z)$ consider the problem of finding for each positive integer $n$ all polynomials $V(z)$ of degree at most $r$ such that the equation

$$
\mathfrak{d}(z) S(z)+V(z) S(z)=0
$$

has a polynomial solution $S(z)$ of degree $n$.

Following the classical terminology we call (1.3) (higher) Heine-Stieltjes spectral problem, polynomial $V(z)$ a (higher) Van Vleck polynomial, and the corresponding polynomial $S(z)$ a (higher) Stieltjes polynomial. To move further we need to formulate two main results of [6], see Corollary 1 and Theorem 9 there.

Proposition 1. For any non-degenerate higher Lamé operator $\mathfrak{d}(z)$ there exist and finitely many (up to a scalar multiple) Stieltjes polynomials $S(z)$ of any sufficiently large degree. Their corresponding Van Vleck polynomials $V(z)$ all have degree exactly $r$.

The next localization result is of a special importance to us.

Theorem 2. For any non-degenerate higher Lamé operator $\mathfrak{d}(z)$ and any $\epsilon>0$ there exists a positive integer $N_{\epsilon}$ such that the zeros of all its Van Vleck polynomials $V(z)$ possessing a Heine-Stieltjes polynomial $S(z)$ of degree $n \geq N_{\epsilon}$ as well as all zeros of these Heine-Stieltjes polynomials belong to Conv $Q_{Q_{k}}^{\epsilon}$. Here Conv $Q_{k}$ is the convex hull of all zeros of the leading coefficient $Q_{k}(z)$ and $\operatorname{Conv}_{Q_{k}}^{\epsilon}$ is its $\epsilon$ neighborhood in the usual Euclidean distance on $\mathbb{C}$.

Remark 1. Proposition 1 together with Theorem 2 show that the zeros of all Van Vleck polynomials having Stieltjes polynomials of degree greater or equal to a sufficiently large positive integer $n$ are confined in some fixed $\epsilon$-neighborhood $\operatorname{Conv}_{Q_{k}}^{\epsilon}$ of $C o n v_{Q_{k}}$. Therefore, there exist plenty of converging subsequences $\left\{\widetilde{V}_{n, i_{n}}(z)\right\}$ of normalized Van Vleck polynomials. Here $\widetilde{V}_{n, i_{n}}(z)$ is a monic polynomial proportional to $V_{n, i_{n}}(z)$ and $V_{n, i_{n}}(z)$ is some Van Vleck polynomial having a Stieltjes polynomial of degree $n$.

It seems natural to pose the following two questions.

Problem 1. What happens with the set $\left\{\widetilde{V}_{n, i}(z)\right\}$ of normalized Van Vleck polynomials having a Stieltjes polynomial of degree exactly $n$ when $n \rightarrow \infty$ ?

Problem 2. What happens with the subsequence $\left\{S_{n, i_{n}}(z)\right\}$ of Stieltjes polynomials whose corresponding sequence $\left\{\widetilde{V}_{n, i_{n}}(z)\right\}$ of normalized Van Vleck polynomials has a limit?

At the present moment we do not have even a conjectural answer to Problem 1. Some initial steps in this direction can be found in [14]. Problem 2 however has a satisfactory answer reported below. 
Definition 1. Given a probability measure $\mu$ supported on some subset of $\mathbb{C}$ we define its Cauchy transform $\mathcal{C}_{\mu}$ as

$$
\mathcal{C}_{\mu}(z)=\int_{\mathbb{C}} \frac{d \mu(\zeta)}{z-\zeta}
$$

Obviously, $\mathcal{C}_{\mu}$ is analytic in the complement to the support of $\mu$.

Definition 2. Given a polynomial $P(z)$ of degree $m$ we define its root-counting measure $\mu_{P}$ as the finite probability measure given by

$$
\mu_{P}(z)=\sum_{j} \frac{k_{j} \delta\left(z-z_{j}\right)}{m}
$$

where $j$ runs over the index set of the set of all distinct zeros $\left\{z_{j}\right\}$ of $P(z), \delta\left(z-z_{j}\right)$ is the usual Dirac delta function concentrated at $z_{j}$ and $k_{j}$ is the multiplicity of the zero $z_{j}$ of $P(z)$.

Assume now that a subsequence $\left\{\widetilde{V}_{n, i_{n}}(z)\right\}$ of normalized Van Vleck polynomials of an operator $\mathfrak{d}(z)$ converges as $n \rightarrow \infty$ to some monic polynomial $\widetilde{V}(z)$ of degree $r$.

Our first result is a far reaching generalization of the Main Theorem of [5] together with the main result of [12] describing the asymptotic root distribution of Heine-Stieltjes polynomials under the asumptions that one picks a sequence with (asymptotically) the same portion of roots in each of the intervals $\left(a_{i}, a_{i+1}\right)$. The latter process corresponds to the choice of a sequence of Van Vlecks converging (up to a scalar factor) to some limiting polynomial.

Theorem 3. For any non-degenerate higher Lamé operator $\mathfrak{d}(z)$ of order $k$ take any subsequence $\left\{\widetilde{V}_{n, i_{n}}(z)\right\}$ of its normalized Van Vleck polynomials converging to some monic polynomial $\widetilde{V}(z)$. Then the sequence $\left\{\mu_{n, i_{n}}\right\}$ of the root-counting measures of the corresponding Stieltjes polynomials $\left\{S_{n, j_{n}}(z)\right\}$ weakly converges to a probability measure $\mu_{\mathfrak{D}, \widetilde{V}}$ whose Cauchy transform $\mathcal{C}_{\mathfrak{o}, \widetilde{V}}(z)$ satisfies almost everywhere in $\mathbb{C}$ the equation

$$
\mathcal{C}_{\mathfrak{d}, \widetilde{V}}^{k}(z)=\frac{\widetilde{V}(z)}{Q_{k}(z)} .
$$

Figures 1 and 2 illustrate the above Theorem 3 .

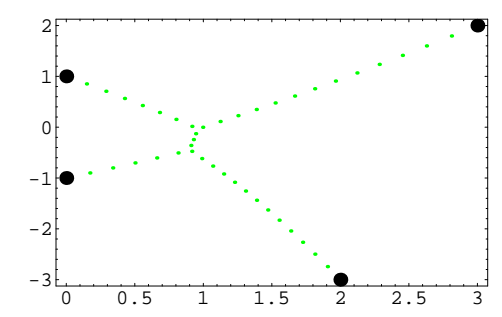

Figure 1. The union of the zeros of 40 linear Van Vlecks for the operator $\frac{d^{3}}{d z^{3}}(Q(z) S(z))+V(z) S(z)=0$ with $Q(z)=\left(z^{2}+1\right)(z-3 I-2)(z+2 I-3)$ whose Stieltjes polynomials are of degree 39 .

Remark 2. Notice that by Theorem 2 the support of $\mu_{\mathfrak{\jmath}, \widetilde{V}}$ should lie within $C o n v_{Q_{k}}$ for any $\widetilde{V}(z)$ which appears as the limit of normalized Van Vleck polynomials. 

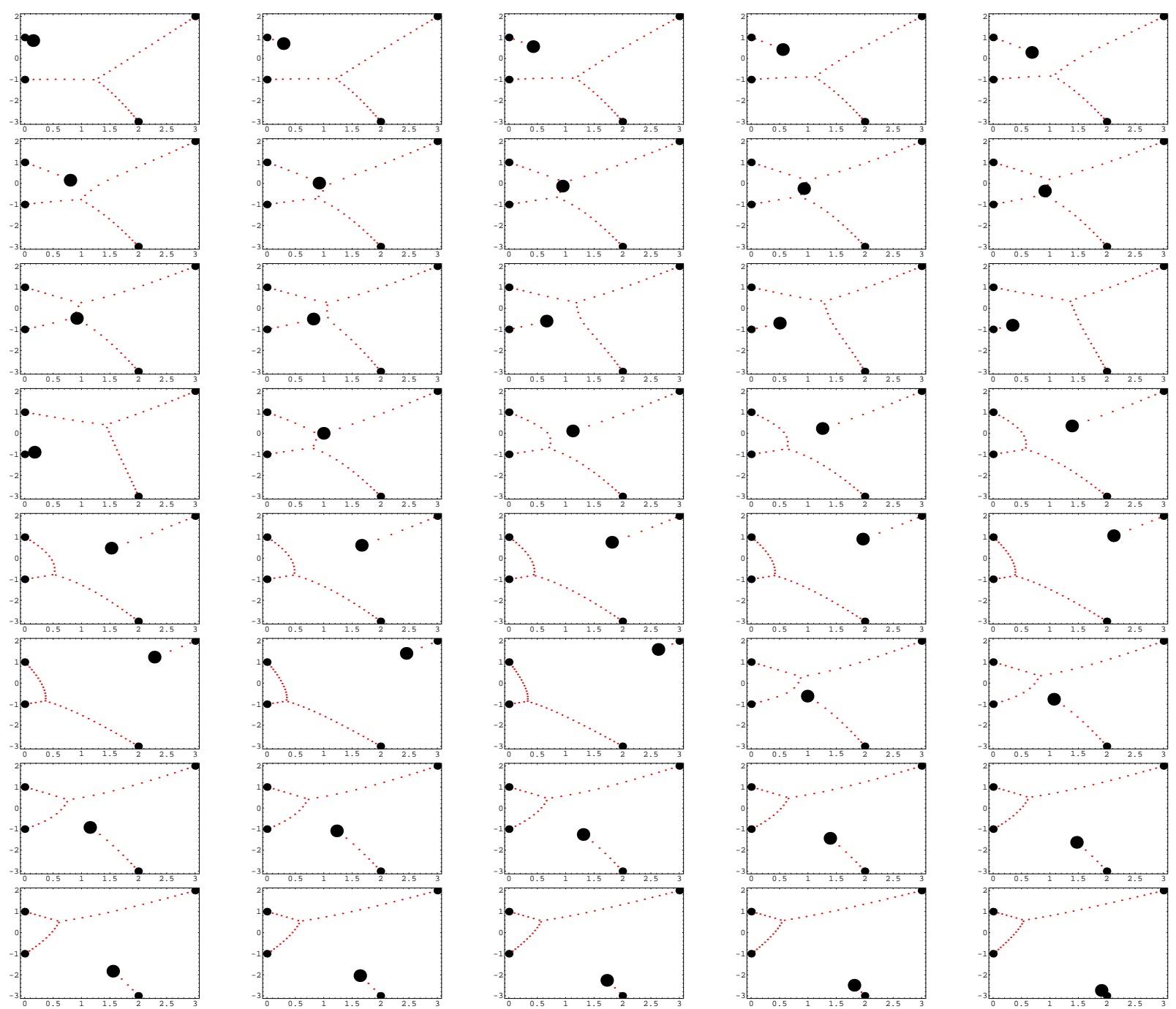

Figure 2. Zeros of 40 different Stieltjes polynomials of degree 39 for the equation $\frac{d^{3}}{d z^{3}}(Q(z) S(z))+V(z) S(z)=0$ with $Q(z)=\left(z^{2}+1\right)(z-3 I-2)(z+2 I-3)$.

Explanation to Figure 2. The smaller dots are the 39 zeros of $S(z) ; 4$ average size dots are the zeros of $Q(z)$ and the single large dot is the (only) zero of the corresponding $V(z)$. For most of the pictures one observes the typical structure of a curvilinear forest with vertices of degrees 1 and 3 only formed by the roots of $S(z)$ which connects the roots of $Q(z)$ and that of $V(z)$. At the same time pictures 3-5 in the second row show the cases of connected support of the corresponding root counting measure.

Our next result describes some properties of a probability measure in case when it satisfies the assumptions of Theorem 3.

Theorem 4. Consider a rational function $R(z)=\frac{V(z)}{Q(z)}$ where $V(z)$ and $Q(z)$ are monic polynomials with $\operatorname{deg} Q(z)-\operatorname{deg} V(z)=k \geq 2$. Assume that the exists a compactly supported probability measure $\mu$ on $\mathbb{C}$ whose Cauchy transform $\mathcal{C}_{\mu}(z)$ 
satisfies almost everywhere (wrt the standard Lebesgue measure on $\mathbb{C}$ ) the equation

$$
\mathcal{C}_{\mu}^{k}(z)=\frac{V(z)}{Q(z)} .
$$

Then such a measure $\mu$ is unique (for a given function $R(z)$ ) and its support is a curvilinear forest with leaves at the roots of $V(z)$ and/or $Q(z)$. This support is straightened out in the local canonical coordinate $w(z)=\int_{z_{0}}^{z} \sqrt[k]{\frac{V(t)}{Q(t)}} d t$.

The next question seems very natural in view of the latter theorem but we do not have even a good guess about its possible answer except for some trivial cases.

Problem 3. Which rational functions $R(z)$ admit a probability measure as in Theorem 4?

Remark 3. In fact we will first prove Theorem 4 and the use it to settle Theorem 3.

Acknowledgements. The authors are sincerely grateful to H. Rullgård for important discussions of the above topic and to Prof. Andrei Martínez-Finkelshtein who brought to our attention the crucial notion of quadratic differential with closed trajectories.

\section{On probability measure With Cauchy transform Whose power is a RATIONAL FUNCTION}

This section is devoted to the proof of Theorem 4. Below we discuss a number of properties of a compactly supported probability measure $\mu$ whose Cauchy transform $\mathcal{C}_{\mu}(z)$ satisfies almost everywhere in $\mathbb{C}$ the equation

$$
\mathcal{C}_{\mu}^{k}(z)=\frac{V(z)}{Q(z)}
$$

where $V(z)$ and $Q(z)$ are monic polynomials with no common factors.

It is clear that $\mathcal{C}_{\mu}(z)$ can have a pole of order at most 1 , which means that $Q(z)$ can only have roots of multiplicity $\leq k$ if $(2.1)$ is satisfied. Suppose now that $Q(z)$ has roots of multiplicity exactly $k$, say $Q(z)=\left(z-a_{1}\right)^{k} \cdot \ldots \cdot\left(z-a_{m}\right)^{k} \widetilde{Q}(z)$, where $\widetilde{Q}(z)$ only has roots of multiplicity $<k$. Taking the $\bar{z}$-derivative in distributional sense yields

$$
\begin{gathered}
\pi \mu=\frac{\partial}{\partial \bar{z}}\left[\frac{1}{\left(z-a_{1}\right) \cdot \ldots \cdot\left(z-a_{m}\right)}\left(\frac{V(z)}{\widetilde{Q}(z)}\right)^{1 / k}\right]= \\
=\sum_{j=1}^{m} \delta_{a_{j}}\left(\frac{V\left(a_{j}\right)}{\widetilde{Q}\left(a_{j}\right)}\right)^{1 / k}+\frac{1}{\left(z-a_{1}\right) \cdot \ldots \cdot\left(z-a_{m}\right)} \frac{\partial}{\partial \bar{z}}\left(\frac{V(z)}{\widetilde{Q}(z)}\right)^{1 / k},
\end{gathered}
$$

where $\delta_{a_{j}}$ is a point mass located at $z=a_{j}$. Proposition 2 below states that the positive measure $\widetilde{\mu}:=\frac{\partial}{\partial \bar{z}}\left(\frac{V(z)}{\bar{Q}(z)}\right)^{1 / k}$ must be supported on a finite union of analytic curves. If $\mathcal{C}_{\mu}^{+}$and $\mathcal{C}_{\mu}^{-}$denotes the one-sided limits of the Cauchy transform as we approach the support of $\mu$, then the Sokhotsky-Plemelj formula tells us that $\tilde{\mu}=\rho(s) d s$ where $\rho(s)=\frac{\left(\mathcal{C}_{\mu}^{+}(s)-\mathcal{C}_{\mu}^{-}(s)\right)}{2} \nu$, with $\nu$ a properly oriented normal of the support, and $d s$ is the usual arc length. Clearly $\rho$ vanishes only at the roots of $V(z)$. From this it immediately follows that the point masses $\delta_{a_{j}}$ can not lie in the closure of the support of $\tilde{\mu}$, since the total mass of $\mu$ is assumed to be finite.

Our goal in this section is to prove that the support of a positive measure $\mu$ whose Cauchy transform satisfies (2.1) is a (curvilinear) forrest. By the above remarks we 
can restrict our attention to measures whose Cauchy transform satisfies (2.1) with $Q(z)$ only having roots of multiplicity $<k$. The reader will easily check the validity of all the arguments given below if we add a finite number of isolated point masses to the measure $\mu$.

Proposition 2. If $\mu$ is a probability measure satisfying (2.1) almost everywhere then the support $\mathfrak{S}_{\mu}$ of $\mu$ is the union of finitely many smooth curve segments, and each of these curves is mapped to a straight line by the (locally defined) mapping

$$
\Psi(z)=\int_{z_{0}}^{z} \sqrt[k]{\frac{V(t)}{Q(t)}} d t
$$

Remark 4. Function $\Psi(z)$ is often referred to as a canonical coordinate. Proof of this Proposition repeats more or less literally that of Lemma 4 in [5] and is included for the sake of completeness. We need the following technical statement.

Lemma 1 (Corollary 2 of [5]). For a finite set $A \subset \mathbb{C}$, a convex domain $U$ and a measurable function $\chi: U \rightarrow A$ the claim that $\frac{\partial \chi}{\partial \bar{z}} \geq 0$ (as a distribution) is equivalent to the existence of real numbers $c_{a}, a \in \AA$ such that $\chi(z)=$ a almost everywhere in $G_{a}$ where

$$
G_{a}=\left\{z \in U ; c_{a}+\Re(a z) \geq c_{b}+\Re(b z) ; \forall b \in A\right\} .
$$

In other words, any (local) domain where $\chi$ attains a constant value is (locally) an angle given by linear inequalities.

Remark 5. Proof of Lemma 1 is based on Corollary 1 of [5] claiming that for a convex domain $U \subset \mathbb{C}$, a finite set $A \subset \mathbb{C}$ and a subharmonic function $v$ defined in $U$ such that $2 \frac{\partial v}{\partial z} \in A$ almost everywhere one has that $v$ coincides with the maximum of a number of linear (non-homogeneous) functions and is, therefore, convex.

Proof of Proposition 2. Let us first prove it in a neighborhood of $z_{0} \in \mathfrak{S}_{\mu}$ which is neither a zero nor a pole of $\frac{V(z)}{Q(z)}$. Choose a branch $B(z)$ of $\sqrt[k]{\frac{V(z)}{Q(z)}}$ in some simply connected neighborhood $\Omega$ of $z_{0}$ and define $\Psi$ as some concrete primitive function of $B(z)$ in $\Omega$. Let $U$ be some small convex neighborhood of $\Psi\left(z_{0}\right)$ such that $\Psi$ maps some neighborhood of $z_{0}$ bijectively on $U$. By (2.1) we can write

$$
C_{\mu}(z)=\chi(\Psi(z)) B(z)
$$

for $z \in \Psi^{-1}(U)$, where $\chi$ takes values in the set of $k$-th roots of unity. Using the variable $w=\Psi(z)$ one gets

$$
\pi \mu=\frac{\partial \mathcal{C}_{\mu}}{\partial \bar{z}}=\frac{\partial \chi(\Psi(z))}{\partial \bar{z}} B(z)=\Psi^{*}\left(\frac{\partial \chi}{\partial \bar{w}}\right) \cdot \frac{\overline{\partial \Psi}}{\partial z} \cdot B(z)=\Psi^{*}\left(\frac{\partial \chi}{\partial \bar{w}}\right) \cdot|B(z)|^{2},
$$

where $\Psi^{*}$ denotes the pullback of distributions defined in $U$ by the map $\Psi$. Since the measure $\mu$ is positive one gets the relation $\frac{\partial \chi}{\partial \bar{w}} \geq 0$ which should be interpreted in the distributional sense. By the above mentioned Corollary $2 U$ is the union of sets $G_{a}$ whose boundaries are finite unions of line segments, such that $\chi$ is constant in each $G_{a}$. Therefore, $\mathfrak{S}_{\mu} \cap \Psi^{-1}(U)=\Psi^{-1}\left(\operatorname{supp} \frac{\partial \chi}{\partial \bar{z}}\right)$ is the union of finitely many curve segments which are mapped to straight lines by $\Psi$.

If $z_{0}$ is a zero or a pole of $\frac{V(z)}{Q(z)}$, we take a disk $D$ centered at $z_{0}$ and not containing any other zeros or poles of $\frac{V(z)}{Q(z)}$.If $\gamma$ is any ray emanating from $z_{0}$ we can define single-valued branches of $\sqrt[k]{\frac{V(z)}{Q(z)}}$ and $\Psi$ in $D \backslash \gamma$. Notice that $\Psi$ is continuous up to $z_{0}$. Let $U$ be any half disc centered at $\Psi\left(z_{0}\right)$ and contained in $\Psi(D \backslash \gamma)$. Then 
by the first part of the proof $\mathfrak{S}_{\mu}$ has the required properties in $\Psi^{-1}(D)$. Varying $\gamma$ and $U$, we see that the same holds in a full neighborhood of $z_{0}$.

Lemma 2. Suppose that the Cauchy transform $\mathcal{C}_{\mu}(z)$ of $\mu$ satisfies (2.1) and $u(z)$ is the logarithmic potential of $\mu$. If $\Psi^{-1}$ is a (locally defined) inverse of $\Psi$ which is a primitive to $\sqrt[k]{\frac{V}{Q}}$, then the function $u \circ \Psi^{-1}$ coincides with the maximum of some number of linear functions and, in particular, is convex (in its domain).

Proof. Since $u$ is subharmonic we need to check that its derivative belongs to a finite set. We prove our lemma in a neighborhood of any $z_{0}$ which is neither a zero of $V(z)$ nor a zero of $Q(z)$. Choose a branch $B(z)$ of $\sqrt[k]{\frac{V(z)}{Q(z)}}$ in some simply connected neighborhood $\Omega$ of $z_{0}$ and define $\Psi(z)$ as some concrete primitive function of $B(z)$ in $\Omega$. Let $U$ be some small convex neighborhood of $\Psi\left(z_{0}\right)$ such that $\Psi(z)$ maps some neighborhood of $z_{0}$ bijectively on $U$. We want to show that $u \circ \Psi^{-1}$ is convex in $U$. By (2.1) we can write

$$
C_{\mu}(z)=\chi(\Psi(z)) B(z)
$$

for $z \in \Psi^{-1}(U)$, where $\chi$ takes values in the set of $k$-th roots of unity. Since $\mathcal{C}_{\mu}(z)=2 \frac{\partial u}{\partial z}$ we have using the variable $w=\Psi(z)$

$2 \frac{\partial}{\partial w} u\left(\Psi^{-1}(w)\right)=2 \frac{\partial u}{\partial z}\left(\Psi^{-1}(w)\right)\left(B\left(\Psi^{-1}(w)\right)\right)^{-1}=\mathcal{C}_{\mu}\left(\Psi^{-1}(w)\right)\left(B\left(\Psi^{-1}(w)\right)\right)^{-1}=\chi(w)$.

Therefore by the above mentioned Corollary 1 the locally defined function $u \circ \Psi^{-1}$ is piecewise linear and convex.

Corollary 1. If an endpoint of any curve segment in the support $\mathfrak{S}_{\mu}$ of $\mu$ is a hanging vertex (i.e. not shared by any other such segment) then this endpoint is either a zero or a pole of $\frac{V(z)}{Q(z)}$, see Figure 2.

Proof. If this were false, then take a point $p$ which is a hanging vertex but not a zero of either $V(z)$ or $Q(z)$. The Cauchy transform $\mathcal{C}_{\mu}$ is supposed to satisfy an algebraic equation whose branching points are exactly the zeroes of $V(z)$ and $Q(z)$. In particular it has no monodromy around $p$. This implies that the limits of $\mathcal{C}_{\mu}$ as we approach $\mathfrak{S}_{\mu}$ from both sides close to $p$ are the same, which in turn implies that $p$ lies off the support of $\mu$.

Proof of Theorem 4 starts with a series of additional observations. Notice that if we fix a branch of $\sqrt[k]{\frac{V(z)}{Q(z)}}$ locally near some point which is neither its root or its pole and consider a multi-valued canonical coordinate

$$
\Psi(z)=\int_{z_{0}}^{z} \sqrt[k]{\frac{V(t)}{Q(t)}} d t
$$

globally i.e. take its full analytic extension then $\Psi(z)$ will be well-defined and univalent only on the universal covering of $\mathbb{C} \backslash(\mathbb{Z}(V) \cup \mathbb{Z}(Q))$ where $\mathbb{Z}(V)$ (resp. $\mathbb{Z}(Q)$ ) is the set of all roots of $V(z)$ (resp. $Q(z)$ ). But due to the existence of a measure $\mu$ we can choose an almost global representative of $\Psi(z)$ on $\mathbb{C}$ substantially reducing its multi-valuedness. Namely, let $\Omega$ be the complement to the support of $\mu$, i.e. $\Omega=\mathbb{C} \backslash \mathfrak{S}_{\mu}$. Define $\Psi^{+}(z)$ in $\Omega$ by

$$
\Psi_{+}(z)=\int_{\mathbb{C}} \log (z-\zeta) d \mu(\zeta)
$$


Obviously, $\Psi_{+}(z)$ is a part of the whole multi-valued function $\Psi(z)$ since for any $z \in \Omega$ one has $\Psi_{+}^{\prime}(z)=\int_{\mathbb{C}} \frac{d \mu(\zeta)}{z-\zeta}=\mathcal{C}_{\mu}(z)$ while $\mathcal{C}_{\mu}(z)$ satisfies $(2.1)$. Since $\Omega$ is never simply-connected the function $\Psi_{+}(z)$ is still multi-valued, namely, going once around some connected component $K$ of $\mathfrak{S}_{\mu}$ in the clockwise direction one increases $\Psi_{+}(z)$ by $2 \pi i \times \mu(K)$, where $\mu(K)>0$ is the mass of the measure $\mu$ concentrated on $K$. Nevertheless the real part $\Re\left(\Psi_{+}(z)\right)$ is a well-defined single-valued function in $\Omega$ coinciding with the logarithmic potential $u(z)$ of $\mu$, namely

$$
u(z)=\Re\left(\Psi_{+}(z)\right)=\int_{\mathbb{C}} \log |z-\zeta| d \mu(\zeta) .
$$

Consider now the family $\Phi$ of curves in $\Omega$ defined by the condition:

$$
\Im\left(\Psi_{+}(z)\right)=\text { const }
$$

which is obviously independent of the choice of the branch of $\Psi_{+}(z)$. One can easily show that the gradient grad $u(z)$ of $u(z)$ coincides with $\overline{\mathcal{C}}_{\mu}(z)$. (Here $\overline{\mathcal{C}}_{\mu}(z)$ is the usual complex conjugate of $\mathcal{C}_{\mu}(z)$.) Thus the family $\Phi$ consists of the integral curves of the vector field $\overline{\mathcal{C}}_{\mu}(z)$ which is well-defined and non-vanishing in $\Omega$. Moving along the trajectories of $\overline{\mathcal{C}}_{\mu}(z)$ in positive time we increase the value of $u(z)$. Finally, for sufficiently large $|z|$ one has

$$
\overline{\mathcal{C}}_{\mu}(z) \simeq \frac{1}{\bar{z}}, \text { and } u(z) \simeq \log |z| .
$$

The next statement is very crucial for the proof of Theorem 4 .

Lemma 3. 1) Any trajectory of $\overline{\mathcal{C}}_{\mu}(z)$ tends when $t \rightarrow+\infty$ either to $\infty$ in $\mathbb{C} P^{1}$ or to a root of $V(z)$.

2) Any trajectory of $-\overline{\mathcal{C}}_{\mu}(z)$ tends to $\mathfrak{S}_{\mu}$ or, in other words, any trajectory of $\overline{\mathcal{C}}_{\mu}(z)$ 'starts' on $\mathfrak{S}_{\mu}$.

Proof. According to lemma 2 locally near any point $p \in \mathfrak{S}_{\mu}$ the logarithmic potential $u$ is given as the maximum of a finite number of linear functions if one uses the canonical coordinate. This shows that the gradient flow points away from the support of $\mu$ everywhere, except possibly at a root of $V(z)$ where the gradient vanishes. At infinity we have $\overline{\mathcal{C}}_{\mu}(z) \approx \frac{1}{\bar{z}}$, which easily implies that the point at infinity is a sink for the flow defined by $\frac{\overline{\mathcal{C}}}{\mu}(z)$. Denote by $K_{\epsilon}$ the Riemann sphere with an $\epsilon$-neighbourhood of $\{\infty\} \cup \mathfrak{S}_{\mu}$ removed. To obtain the proof we need to show that a trajectory cannot stay in a subset of $K_{\epsilon}$ for any $\epsilon>0$ when $t \rightarrow+\infty$. By construction any such $K_{\epsilon}$ does not contain singular points of $\overline{\mathcal{C}}_{\mu}(z)$. Thus, we only need to rule out the occurrence of closed trajectories and recurrence in $K_{\epsilon}$. But both of these possibilities are indeed forbidden by the fact that $\overline{\mathcal{C}}_{\mu}(z)$ is the gradient field of a function without singularities in $K_{\epsilon}$ for any $\epsilon>0$.

Definition 3. A trajectory of $\overline{\mathcal{C}}_{\mu}(z)$ ending at a root of $V(z)$ is called exceptional.

Lemma 4. There exists at most a finite number of trajectories of $\overline{\mathcal{C}}_{\mu}(z)$ ending at a given root of $V$.

Proof. Wlog assume that a root of $V(z)$ lies at the origin and has a multiplicity $p$. Notice that

$$
\Im\left(\Psi_{+}(z)\right)=\Im \int_{0}^{z} \sqrt[k]{\frac{V(t)}{Q(t)}} d t .
$$


We now have $\sqrt[k]{\frac{V(z)}{Q(z)}}=z^{p / k}\left(a_{p}+a_{p+1} z+\ldots\right)^{1 / k}$. Choosing a single-valued branch of $\left(a_{p}+a_{p+1} z+\ldots\right)^{1 / k}$, say $b_{0}+b_{1} z+\ldots$, and taking $\xi=z\left(b_{0}+b_{1} z+\ldots\right)^{k / p}$, which can be chosen single-valued in a neighbourhood of the origin, we get $\sqrt[k]{\frac{V(z)}{Q(z)}}=\xi^{p / k}$ which is valid in a neighbourhood of the origin minus the slit defined by the support of our measure $\mu$. Integration yields

$$
\int_{0}^{z} \sqrt[k]{\frac{V(t)}{Q(t)}} d t=\left(\frac{p+k}{k}\right) \xi^{(p+k) / k}
$$

Since there is only a finite number of rays in the $\xi$-plane entering the origin along which $\Im\left(\xi^{(p+k) / k}\right) \equiv 0$, our claim follows.

Remark 6 . The proof of the previous lemma copies some of local studies of zeroes of quadratic differentials, see [16], Ch.2. It is indeed possible to interpret the support of $\mu$ as a geodesic of a higher order differential, which in turn allows us to state more explicitley exactly how many exceptional trajectories enter a hanging vertex of the support of $\mu$. However we postpone detailed study of this topic, see [9].

Definition 4. We denote the union of $\mathfrak{S}_{\mu}$ and all exceptional trajectories by $\Upsilon_{\mu}$ and call it the extended support of $\mu$.

The following statement is very essential.

Lemma 5. The extended support $\Upsilon_{\mu}$ is topologically a tree.

Proof. Indeed, by lemmas 3 and 4 the flow on $\mathbb{C P}^{1} \backslash \Upsilon_{\mu}$ defined by the gradient vector field $\bar{C}_{\mu}(z)$ (which is non-vanishing there) contracts the whole domain to the point at infinity. The result follows.

Let $\mu$ be a measure whose Cauchy transform satisfies (2.1) and denote $\Omega^{*}=$ $\mathbb{C} \backslash \Upsilon_{\mu}$. Define now the following specialization of the function $\Psi_{+}(z)$ to $\Omega^{*}$ :

$$
\Psi_{++}(z)=\int_{\mathbb{C}} \log (z-\zeta) d \mu(\zeta)
$$

where $z \in \Omega^{*}$. Note that $\Psi_{++}(z)$ is multi-valued only up to addition of multiples of $2 \pi i$.

Lemma 6. The function $\Psi_{++}(z)$ determines a mapping of $\Omega^{*}$ onto a domain $H=\{w \mid \Re w>h(\Im w)\}$ where $h$ is a picewise-continuous function. Moreover, $\Psi_{++}^{-1}: H \rightarrow \Omega^{*}$ is a single-valued function, see Fig. 3 .

Proof. We have already noticed that $\Psi_{++}(z)$ is defined up to a multiple of $2 \pi i$ in $\Omega^{*}$ and that $\Psi_{++}^{\prime}(z)=\mathcal{C}_{\mu}(z)$. If $\gamma$ is a small curve segment of $\operatorname{supp}(\mu)$ and if $U$ is a small one sided neighbourhood of $\gamma$, then Proposition 2 states that $\gamma$ is mapped to a straight line segment by $\Psi_{++}$which is not horizontal and that $U$ is mapped to the right of this straight segment. The latter follows if we observe that in the proof of Proposition 2 we have $\chi=1$ in $U$ and $\Re(\chi) \leq 1$ everywhere. If $\gamma$ is a part of an exceptional trajectory then it is mapped by $\Psi_{++}$to a straight horizontal line segment since exceptional trajectories are level curves of $\Im\left(\Psi_{++}\right)$. Continuing $\Psi_{++}$around $\Upsilon_{\mu}$ we obtain a broken piecewise linear curve of the form $\{\Re(w)=h(\Im(w))\}$ bounding a domain $H$ of the form $\{\Re(w)>h(\Im(w))\}$ where $h$ is a piecewise continuous function. It is clear that $\Psi_{++}$maps $\Omega$ onto $H$ with boundary to boundary. The function $\psi(z)=\exp \left(-\Psi_{++}(z)\right)$ is single-valued on $\Omega^{*}$ 
and maps $\Omega^{*} \cup\{\infty\}$ to $D=\{\zeta: \log |\zeta|<-h(-\arg \zeta)\}$, does not vanish in $\Omega^{*}$ and has a simple zero at infinity. It follows that $\psi$ is bijective on $\Omega^{*} \cup\{\infty\} \rightarrow D$ and hence $\Psi_{++}^{-1}(w)=\psi^{-1}\left(e^{-w}\right)$ is single-valued. This concludes the proof of the lemma.
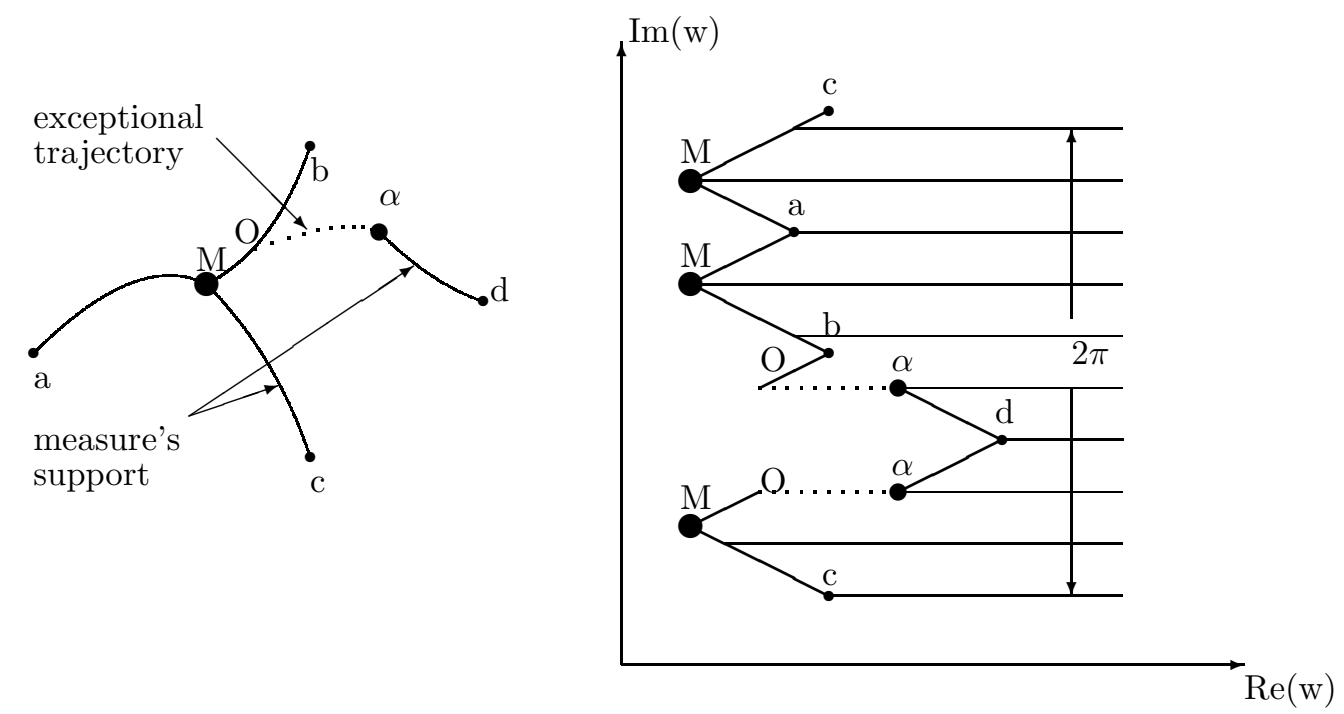

Figure 3. Extended support $\Upsilon_{\mu}$ of a positive measure associated with a rational function $\frac{z-\alpha}{(z-a)(z-b)(z-c)(z-d)}$ and its image under the mapping $\Psi_{++}(z)$.

Explanation to Figure 3. The left picture shows the support of a measure $\mu$ consisting of 2 components - one connecting three poles $a, b, c$ and the other connecting the only zero $\alpha$ with the remaining pole $d$. The exceptional trajectory connects the point $O$ on the first component with the zero $\alpha$ so that the extended support $\Upsilon_{\mu}$ forms a tree. The map $\Psi_{++}(z)$ wraps out the complement $\mathbb{C} \backslash \Upsilon_{\mu}$ onto the strip bounded by two horizontal lines and the piecewise linear curve shown on the right picture. This map has a period $2 \pi \sqrt{-1}$. We go around the extended support in the anti-clockwise direction starting from the pole $c$. The piecewise linear curve on the right picture shows the images of the special points we meet while doing this: $c, M, O, \alpha, d, \alpha, O, b, M, a, M$ and returning back to $c$ but with the imaginary part increased by $2 \pi$. Each time we meet one of the poles or the point $M$ we have to change our direction by $\pm 120^{\circ}$.

We can finally prove the uniqueness of the required measure. Indeed, assume that there are two different probability measures $\mu_{1}$ and $\mu_{2}$ whose Cauchy transforms solve (2.1) almost everywhere and let $u_{1}(z)$ and $u_{2}(z)$ be their logarithmic potentials. Notice that there is only one branch of $\sqrt[k]{\frac{V(z)}{Q(z)}}$ which has $\frac{1}{z}$ as its asymptotics near $\infty$ in $\mathbb{C} P^{1}$. Therefore the Cauchy transforms and logarithmic potentials of $\mu_{1}$ and $\mu_{2}$ have to coincide in some neighborhood of $\infty$. We will show that $u_{2}(z) \geq u_{1}(z)$ in $\Omega_{1}^{*}=\mathbb{C} \backslash \Upsilon_{\mu, 1}$ and $u_{1}(z) \geq u_{2}(z)$ in $\Omega_{2}^{*}=\mathbb{C} \backslash \Upsilon_{\mu, 2}$. Indeed, $u_{1}\left(\Psi_{++}^{-1}(w)\right)=\Re(w)$ for all $w \in H_{1}$ and $u_{2}\left(\Psi_{++}^{-1}(w)\right)=\Re(w)$ for all sufficiently large $\Re(w)$. On the other hand, $u_{2} \circ \Psi_{++}^{-1}$ is piecewise linear and convex on any ray $\Im(w)=$ const in $H_{1}$. Therefore, $u_{2}\left(\Psi_{++}^{-1}(w)\right) \geq \Re(w)$ for all $w \in H_{1}$. Changing place of $u_{1}(z)$ and $u_{2}(z)$ we get the second inequality. Since $u_{1}(z)$ and $u_{2}(z)$ are continuous in the whole $\mathbb{C}$ they should coincide. But the measures $\mu_{1}$ and $\mu_{2}$ are obtained as $\Delta u_{1}(z)$ and $\Delta u_{2}(z)$, therefore they coincide as well. 
We finish this section with some information about connected components of $\mathfrak{S}_{\mu}$.

Lemma 7. Each connected component of $\mathfrak{S}_{\mu}$ contains either some number of roots of $Q(z)$ and equally many roots of $V(z)$ or it contains $k+j$ roots of $Q(z)$ and $j$ roots of $V(z)$ for some $j=0, \ldots, r$. (We count roots with multiplicities here.)

Proof. Going around such a component should result in a trivial monodromy of the branches of $\sqrt[k]{\frac{V(z)}{Q(z)}}$. Notice that these branches are cyclicly ordered and a small clockwise oriented loop around a root of $V(z)$ results in the cyclic clockwise shift by $\frac{2 \pi}{k}$ while a small clockwise oriented loop around a root of $Q(z)$ results in the cyclic counterclockwise shift by $\frac{2 \pi}{k}$.

\section{Proving Theorem 3}

Our scheme follows roughly the scheme suggested in [5]. We need to prove under its assumptions the sequence $\left\{\mu_{n, i_{n}}\right\}$ of root-counting measures of the Stieltjes polynomials $\left\{S_{n, i_{n}}(z)\right\}$ converges weakly to a probability measure $\mu_{\mathfrak{d}(z), \tilde{V}}$ whose Cauchy transform $\mathcal{C}_{\mathfrak{d}(z), \widetilde{V}}(z)$ satisfies almost everywhere in $\mathbb{C}$ the equation (1.4).

To simplify the notation we denote by $\left\{\bar{S}_{n}(z)\right\}$ the chosen sequence $\left\{S_{n, i_{n}}(z)\right\}$ of Stieltjes polynomials whose normalized Van Vleck polynomials $\left\{\widetilde{V}_{n, i_{n}}(z)\right\}$ converge to $\widetilde{V}(z)$ and let $\left\{\bar{\mu}_{n}\right\}$ denote the sequence of its root-counting measures. Also let $\bar{\mu}_{n}^{(i)}$ be the root measure of the $i$ th derivative $\bar{S}_{n}^{(i)}(z)$. Assume now that $N N$ is a subsequence of natural numbers such that

$$
\bar{\mu}^{(j)}=\lim _{n \rightarrow \infty, n \in N N} \bar{\mu}_{n}^{(j)}
$$

exists for all $j=0,1, \ldots, k$. The next lemma shows that the Cauchy transform of $\bar{\mu}=\bar{\mu}^{(0)}$ satisfies the required algebraic equation.

Lemma 8. The measures $\bar{\mu}^{j}$ are all equal and the Cauchy transform $\mathcal{C}(z)$ of their common limit satisfies the equation (1.4) for almost every $z$.

Proof. We have

$$
\frac{\bar{S}_{n}^{(j+1)}(z)}{(n-j) \bar{S}_{n}^{(j)}(z)} \rightarrow \int \frac{d \bar{\mu}^{(j)}(\zeta)}{z-\zeta}
$$

with convergence in $L_{l o c}^{1}$, and by passing to a subsequence again we can assume that we have pointwise convergence almost everywhere. From the relation

$$
\mathfrak{d}(z) \bar{S}_{n}(z)+V_{n}(z) \bar{S}_{n}(z)=0
$$

it follows that

$\frac{Q_{k}(z) \bar{S}_{n}^{(k)}(z)}{n \ldots(n-k+1) \bar{S}_{n}(z)}+\frac{V_{n}(z)}{n \ldots(n-k+1)}=-\sum_{l=0}^{k-1} \frac{Q_{l}(z)}{(n-l) \ldots(n-k+1)}\left(\prod_{j=0}^{l-1} \frac{\bar{S}_{n}^{(j+1)}(z)}{(n-j) \bar{S}_{n}^{(j)}(z)}\right)$.

One can immediately check that $-\frac{V_{n}(z)}{n(n-1) \ldots(n-k+1)} \rightarrow \widetilde{V}(z)$, while the sum in the right-hand side converges pointwise to 0 almost everywhere in $\mathbb{C}$ due to presence of the factors $(n-l) \ldots(n-k+1)$ in the denominators. Thus, for almost all $z \in \mathbb{C}$ one has

$$
\frac{\bar{S}_{n}^{(k)}(z)}{n(n-1) \ldots(n-k+1) \bar{S}_{n}(z)} \rightarrow \frac{\widetilde{V}(z)}{Q_{k}(z)}
$$


when $n \rightarrow \infty$ and $n \in N N$. If $u^{(j)}(z)$ denotes the logarithmic potential of $\bar{\mu}^{(j)}$, then one has

$$
\begin{gathered}
u^{(k)}(z)-u^{(0)}(z)=\lim _{n \rightarrow \infty} \frac{1}{n} \log \left|\frac{\bar{S}_{n}^{(k)}(z)}{n(n-1) \ldots(n-k+1) \bar{S}_{n}(z)}\right|= \\
=\lim _{n \rightarrow \infty} \frac{1}{n}\left(\log |\tilde{V}(z)|-\log \left|Q_{k}(z)\right|\right)=0 .
\end{gathered}
$$

On the other hand we have that $u^{(0)}(z) \geq u^{(1)}(z) \geq \ldots \geq u^{(k)}(z)$, see Lemma 9 below. Hence all the potentials $u^{(j)}(z)$ are equal, and all $\mu_{j}=\Delta u^{(j)} / 2 \pi$ are equal as well. Finally we get

$$
C^{k}(z)=\lim _{n \rightarrow \infty} \Pi_{j=0}^{k-1} \frac{\bar{S}_{n}^{(j+1)}(z)}{(n-j) \bar{S}_{n}^{(j)}(z)}=\lim _{n \rightarrow \infty} \frac{\bar{S}_{n}^{(k)}(z)}{n \ldots(n-k+1) \bar{S}_{n}(z)}=\frac{\widetilde{V}(z)}{Q_{k}(z)}
$$

for almost all $z$. This completes the proof of the existence of a compactly supported probability measure whose Cauchy transform satisfies $(1.4)$ in the case when $\widetilde{V}(z)$ is the limit of a sequence of normalized Van Vleck polynomials of a Heine-Stieltjes problem with the leading coefficient of the operator equal to $Q_{k}(z)$. The uniqueness of such a measure was obtained in $\S 2$.

Lemma 9 (see Lemma 8 of $[5]$ ). Let $\left\{p_{m}(z)\right\}$ be a sequence of polynomials, such that $n_{m}:=\operatorname{deg} p_{m} \rightarrow \infty$ and there exists a compact set $K$ containing the zeros of all $p_{m}(z)$ simultaneously. Finally, let $\mu_{m}$ and $\mu_{m}^{\prime}$ be the root-counting measures of $p_{m}(z)$ and $p_{m}^{\prime}(z)$ resp. If $\mu_{m} \rightarrow \mu$ and $\mu_{m}^{\prime} \rightarrow \mu^{\prime}$ with compact support and $u(z)$ and $u^{\prime}(z)$ are the logarithmic potentials of $\mu$ and $\mu^{\prime}$, then $u^{\prime}(z) \leq u(z)$ in the whole $\mathbb{C}$. Moreover, $u(z)=u^{\prime}(z)$ in the unbounded component of $\mathbb{C} \backslash \operatorname{supp} \mu$.

Proof. Assume wlog that $p_{m}(z)$ are monic. Let $K$ be a compact set containing the zeros of all $p_{m}(z)$. We have

$$
u(z)=\lim _{m \rightarrow \infty} \frac{1}{n_{m}} \log \left|p_{m}(z)\right|
$$

and

$$
u^{\prime}(z)=\lim _{m \rightarrow \infty} \frac{1}{n_{m}-1} \log \left|\frac{p_{m}^{\prime}(z)}{n_{m}}\right|=\lim _{m \rightarrow \infty} \frac{1}{n_{m}} \log \left|\frac{p_{m}^{\prime}(z)}{n_{m}}\right|
$$

with convergence in $L_{l o c}^{1}$. Hence

$$
u^{\prime}(z)-u(z)=\lim _{m \rightarrow \infty} \frac{1}{n_{m}} \log \left|\frac{p_{m}^{\prime}(z)}{n_{m} p_{m}(z)}\right|=\lim _{m \rightarrow \infty} \frac{1}{n_{m}} \log \left|\int \frac{d \mu_{m}(\zeta)}{z-\zeta}\right| .
$$

Now, if $\phi(z)$ is a positive test function it follows that

$$
\begin{gathered}
\int \phi(z)\left(u^{\prime}(z)-u(z)\right) d \lambda(z)=\lim _{m \rightarrow \infty} \int \phi(z) \log \left|\int \frac{d \mu_{m}(\zeta)}{z-\zeta}\right| d \lambda(z) \leq \\
\leq \lim _{m \rightarrow \infty} \int \phi(z) \int \frac{d \mu_{m}(\zeta)}{|z-\zeta|} d \lambda(z) \leq \lim _{m \rightarrow \infty} \iint \frac{\phi(z) d \lambda(z)}{|z-\zeta|} d \mu_{m}(\zeta)
\end{gathered}
$$

where $\lambda$ denotes Lebesgue measure in the complex plane. Since $\frac{1}{|z|}$ is locally integrable, the function $\int \phi(z)|z-\zeta|^{-1} d \lambda(z)$ is continuous, and hence bounded by a constant $M$ for all $z$ in $K$. Since supp $\mu_{m} \in K$, the last expression in the above inequality is bounded by $M / n_{m}$, hence the limit when $m \rightarrow \infty$ equals to 0 . This proves $u^{\prime}(z) \leq u(z)$.

In the complement to supp $\mu, u(z)$ is harmonic and $u^{\prime}(z)$ is subharmonic, hence $u^{\prime}(z)-u(z)$ is a negative subharmonic function. Moreover, in the complement of $K, p_{n}^{\prime}(z) /\left(n_{m} p_{m}(z)\right)$ converges uniformly on compact sets to the Cauchy transform $\mathcal{C}_{\mu}(z)$ of $\mu$. Since $\mathcal{C}_{\mu}(z)$ is a non-constant holomorphic function in the unbounded component of $\mathbb{C} \backslash K$, then by the above $u^{\prime}(z)-u(z)=0$ there. By the maximum 
principle for subharmonic functions it follows that $u^{\prime}(z)-u(z)=0$ holds in the unbounded component of $\mathbb{C} \backslash \operatorname{supp} \mu$ as well.

To accomplish the proof of Theorem 3 we need to show that we have the convergence for the whole sequence and not just for some subsequence. Assume now that the sequence $\bar{\mu}_{n}$ does not converge to $\bar{\mu}$. Then we can find a subsequence $N N^{\prime}$ such that $\bar{\mu}_{n}$ stay away from some fixed neighborhood of $\bar{\mu}$ in the weak topology, for all $n \in N N^{\prime}$. Again by compactness, we can find a subsequence $N N^{*}$ of $N N^{\prime}$ such that all the limits for root measures for derivatives exist for $j=0, \ldots, k$. But then $\bar{\mu}^{(0)}$ must coincide with $\bar{\mu}$ by the uniqueness and the latter lemma. We get a contradiction to the assumption that $\mu_{n}$ stay away from $\bar{\mu}$ for all $n \in N N^{\prime}$ and hence all $n \in N N$.

\section{FinAL REMARKS}

As an observant reader easily notices the present paper leads to many more questions than it provides answers to, some of those already mentioned in the introduction. We use this circumstance as an excuse for having this lengthy final section.

I. Call the differential monomial $Q_{k}(z) \frac{d^{k}}{d z^{k}}$ the leading term of a differential operator $\mathfrak{d}(z)=\sum_{i=1}^{k} Q_{i}(z) \frac{d^{i}}{d z^{i}}$. Our first conjecture states that asymptotically Van Vleck and Stieltjes polynomials of the equation (1.3) behave very similarly to that of the equation

$$
Q_{k}(z) \frac{d^{k} S(z)}{d z^{k}}+V(z) S(z)=0
$$

Let $\mathrm{Pol}_{r}$ denote the space of all monic polynomials of degree $r$. Take a nondegenerate $\mathfrak{d}(z)$. For some Van Vleck polynomial $V(z)$ of $\mathfrak{d}(z)$ denote by $\widetilde{V}(z)$ its monic scalar multiple. For a given positive integer $n$ denote by $\left\{V_{n, i}(z)\right\}$ the set of all Van Vleck polynomials $V(z)$ whose Stieltjes polynomials have degree exactly $n$. (Each $V(z)$ is repeated as many times as its multiplicity prescribes, see [6]) Notice that for sufficiently large $n$ the set $\left\{V_{n, i}(z)\right\}$ belongs to $\operatorname{Pol}_{r}$, i.e. each Van Vleck has degree exactly $r$. Transform now the set $\left\{V_{n, i}(z)\right\}$ into a finite measure $\sigma_{n}(\mathfrak{d}(z))$ in $\mathrm{Pol}_{r}$ by assigning to each element the finite mass equal to the inverse of the cardinality of $\left\{V_{n, i}(z)\right\}$.

Conjecture 1. For any non-degenerate $\mathfrak{d}(z)$

- the sequence $\sigma_{n}(\mathfrak{d}(z))$ converges weakly to a measure $\Sigma(\mathfrak{d}(z))$ compactly supported in $\mathrm{Pol}_{r}$

- the measure $\Sigma(\mathfrak{d}(z))$ depends only on the leading monomial $Q_{k}(z) \frac{d^{k}}{d z^{k}}$ of $\mathfrak{d}(z)$.

Remark 7. One can defined the natural 'projection' of the finite set of polynomials $\sigma_{n}(\mathfrak{d}(z))$ to the union of the zero loci $\mathfrak{Z}\left(\sigma_{n}(\mathfrak{d}(z))\right)$ of these polynomials and then turn the latter set into a finite measure in the same way. Conjecture 1 implies that this set of finite measures converges to the standard measure supported inside $\operatorname{Conv}_{Q_{k}}$, see two left pictures on Fig. 4.

II. Let us present a quite surprising (at least to us) observation about the asymptotic distribution of Van Vleck versus Stieltjes polynomials obtained through numerical experiments. We state it first in the simplest case $r=1$. Notice that for any nondegenerate Lamé operator $\mathfrak{d}(z)=Q_{k}(z) \frac{d^{k-1}}{d z^{k-1}}+\ldots$ of order $k$ with $r=1$ and sufficently large $n$ there exist exactly $(n+1)$ Van Vleck polynomials all having 
degree 1, see Proposition 1. Denote by $\xi_{n}$ the root-counting measure for the union of all zeros of these polynomials, and define $\xi=\lim _{n \rightarrow \infty} \xi_{n}$ (if it exists). Assuming that $\xi$ exists which is strongly supported by our numerics we formulate the following fact illustrated on Figure 4 below.

Observation 1. The support of $\xi$ has a very surprising topological and geometric resemblance with that for the limiting root counting measure of the sequence of eigenpolynomials for the exactly solvable (i.e. $r=0$ ) operator $T=Q_{k}(z) \frac{d^{k}}{d z^{k}}$, see Theorem 3 of [5]. (Notice that in case $r=0$ eigenpolynomials are natural analogs of Heine-Stieltjes polynomials for such an operator.)
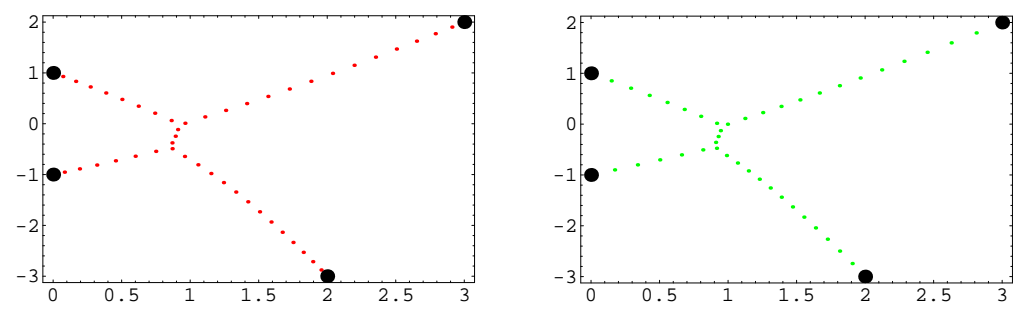

Figure 4. The zeros of the eigenpolynomial of degree 40 for the operator $Q(z) \frac{d^{4}}{d z^{4}}$ (left) and combined zeros of the 40 linear Van Vlecks having a Stieltjes polynomial of degree 39 for the operator $\frac{d^{3}}{d z^{3}}(Q(z) S(z))+V(z) S(z)=0$ with $Q(z)=\left(z^{2}+1\right)(z-3 I-2)(z+2 I-3)$, comp. Fig 1 .

This resemblance seems to persist for larger $r$. Namely, consider two higher Lamé operators $\mathfrak{d}_{1}$ and $\mathfrak{d}_{2}$ of the form

$$
\mathfrak{d}_{1}=Q_{k}(z) \frac{d^{l}}{d z^{l}}+\ldots \text { and } \mathfrak{d}_{2}=Q_{k}(z) \frac{d^{l-1}}{d z^{l-1}}+\ldots
$$

where $\operatorname{deg} Q_{k}(z) \geq k$ and $l \leq k$. Let $\bar{V}_{n}(z)$ denote the product of all Van Vleck polynomials having Stieltjes polynomials of degree $n$ for $\mathfrak{d}_{2}$ and let $\bar{S}_{n}(z)$ denote the product of all Stieltjes polynomials of degree $n$ for $\mathfrak{d}_{1}$. Let $\nu_{1}$ be the asymptotic root-counting measure for the sequence $\left\{\bar{S}_{n}(z)\right\}$ and $\nu_{2}$ be the asymptotic rootcounting measure for the sequence $\left\{\bar{V}_{n}(z)\right\}$ (if they exist).

Observation 2. In the above notation the supports of $\nu_{1}$ and $\nu_{2}$ have surprising geometric and topological similarities, see Figure 5.

Problem 4. Find an explanation for the above observations.

III. Curves similar to that in the support of $\mu_{\mathfrak{d}(z), \tilde{V}}$ appeared earlier as Stokes lines of linear differential equations with polynomial coefficients.

Problem 5. What is the exact relation between the WKB-theory for the equation

$$
y^{(k)}+\frac{\tilde{V}(z)}{Q(z)} y=0
$$

and the asymptotic root counting measure $\mu_{\mathfrak{d}(z), \tilde{V}}$ of the sequence of Stieltjes polynomials with the sequence of normalized Van Vleck polynomials converging to $\tilde{V}$ ?

In the case of a generalized Lamé equation (1.1) one at least has a strict definition of the global Stokes line for such an equation, see e.g. [7] and the support of the corresponding $\mu_{\mathfrak{d}(z), \tilde{V}}$ coincides with a part of this global Stokes line. Even in this 

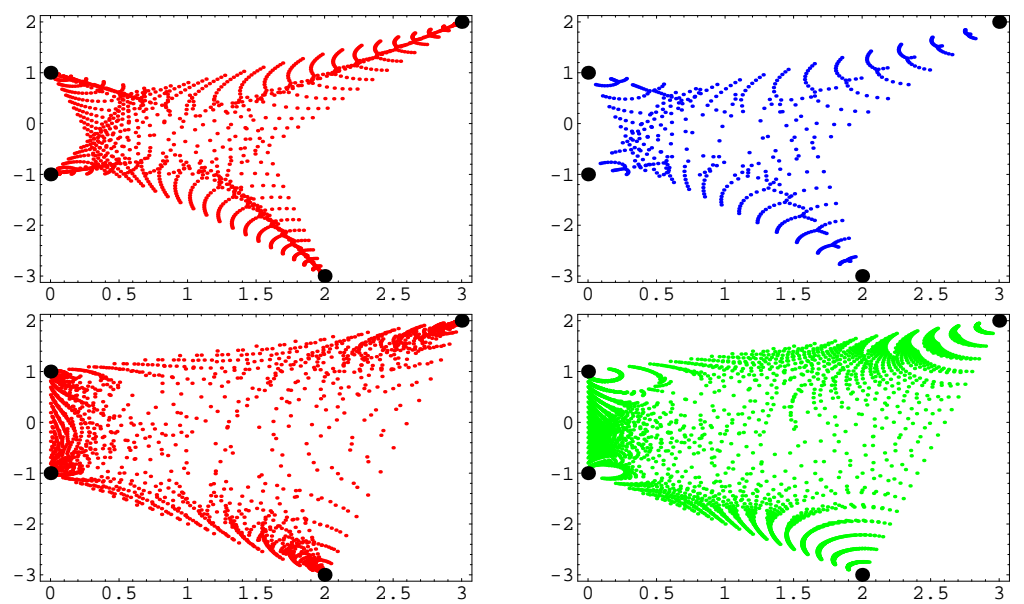

Figure 5. Combined zeros of the Stieltjes polynomials for $Q(z) \frac{d^{3}}{d z^{3}}$ and $Q(z) \frac{d^{2}}{d z^{2}}$ (two left pics) and combined zeros of the Van Vleck polynomials for $Q(z) \frac{d^{2}}{d z^{2}}$ and $Q(z) \frac{d}{d z}$ with $Q(z)=\left(z^{2}+1\right)(z-3 I-2)(z+2 I-3)$ as described in Observation 2 above. Notice the resemblance of shapes!

classical case there is an interesting problem of defining what part of the global Stokes line is covered by this support.

For the case of equations of order exceeding 2 the situation is much worse since even a good definition of the global Stokes line creates serious problems, see e.g. $[2],[3]$, references therein and further publications of the same authors.

IV. Next question generalizes Problem 3 from the introduction.

Problem 6. For what plane algebraic curves in $\mathbb{C}^{2}$ with the given coordinate system $(z, w)$ there exists a compactly supported probability measure whose Cauchy transform is a section of this algebraic curve almost everywhere in $\mathbb{C}$ ?

At the moment the authors know of several such classes of algebraic curves related to eigenpolynomials of linear ode, but it is completely unclear how to describe the whole set of such curves.

V. In [4] T. Bergkvist obtained a number of interesting results and conjectures in the case of degenerate exactly solvable operators, i.e $\operatorname{deg} Q_{k}<k+r$, see Introduction. Motivated by her results we formulate the following conjecture and a question.

Conjecture 2. For any degenerate Lamé operator and any positive integer $N_{0}$ the union of all the roots to polynomials $V$ and $S$ taken over $\operatorname{deg} S \geq N_{0}$ is always unbounded. Therefore, this property is a key distinction between non-degenerate and degenerate Lamé operators.

Problem 7. Extend the results of this paper to the case of degenerate higher Lamé operators.

VI. Numerical experiments suggest that Stieltjes polynomials of consecutive degrees show a stable root-interlacing pattern along the curves in $\mathfrak{S}_{\mu}$ where $\mu$ is the corresponding asymptotic root counting measure. This phenomenon is especially easy to explain and illustrate in the case of exactly solvable operators, i.e. $r=0$.

Conjecture 3. For any exactly solvable operator $\mathfrak{d}(z)$ the family $\left\{S_{n}(z)\right\}_{n \in \mathbb{N}, n \geq n_{0}}$ of its eigenpolynomials ( $\operatorname{deg} S_{n}(z)=n$ for $n \in \mathbb{N}$ ) has the interlacing property along the support of its asymptotic root counting measure. In other words, the zeros of 
any two consecutive polynomials $S_{n+1}(z)$ and $S_{n}(z)$ interlace along every curve segment in $\mathfrak{S}_{\mu}$ for all sufficiently high degrees $n$.

Some caution is required when defining the notion of interlacing since a) the zeros of $S_{n}(z)$ do not lie exactly on $\mathfrak{S}_{\mu}$ and b) $\mathfrak{S}_{\mu}$ has a nontrivial topological structure. One has to remove sufficiently small neighborhoods of the singular points of $\mathfrak{S}_{\mu}$ where several smooth branches meet and to project the zeros of $S_{n}(z)$ onto $\mathfrak{S}_{\mu}$ along some fixed in advance normal bundle to the smooth part of $\mathfrak{S}_{\mu}$.

An example illustrating the interlacing phenomenon conjectured above is shown on Figure 6.

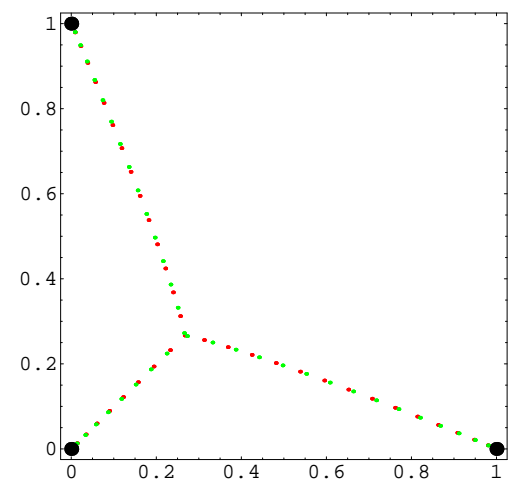

Figure 6. Interlacing pattern for the roots of $S_{40}(z)$ and $S_{41}(z)$ which are the eigenpolynomials of the operator $z(z-1)(z-I) \frac{d^{3}}{d z^{3}}$.

Similar interlacing property was observed for any family of Stieltjes polynomials of increasing degree as soon as the sequence of their normalized Van Vleck polynomials has a limit. Some results about interlacing can be found in [1].

VII. In connection with the classical Bochner-Krall problem which asks to describe all families of orthogonal polynomials appearing as the families of eigenpolynomials for linear differential operators with polynomial coefficients it is natural to ask the following.

Problem 8. Describe all Lamé operators for which all their Van Vleck and Stieltjes polynomials have only real roots.

VIII. Finally theorem 3 can be reinterpreted as the statement that the level curves of the logarithmic potential $u_{\mu}(z)$ of the measure $\mu_{\tilde{V}}$ which we construct in this theorem are trajectories of the differential $(\sqrt{-1})^{k} \frac{\widetilde{V}(z)}{Q_{k}(z)} d z^{k}$ of order $k$. Since almost all level curves of $u_{\mu}(z)$ are closed curve we are tempted to call the latter differential Strebel. This makes perfect sense when $k=2$, see [14] and [11]. But for $k>2$ even a definition of a Strebel differential is so far missing.

Problem 9. Define a notion of a Strebel (rational) differential of order $k>2$.

\section{REFERENCES}

[1] A. Agnew, A. Bourget, T. McMillen, On the zeros of complex Van Vleck polynomials, J. Comp. Appl. Math, 223, (2009), 862-871.

[2] T. Aoki, T. Kawai and Y. Takei, New turning points in the exact WKB analysis for higher order ordinary diffrential equations, Analyse algébrique des perturbations singuliéres. I, Hermann, (1994), 69-84. 
[3] T. Aoki, T. Kawai and Y. Takei, On the exact WKB analysis for the third order ordinary differential equations with a large parameter, Asian J. Math., vol 2, (1998), 625-640.

[4] T. Bergkvist, On asymptotics of polynomial eigenfunctions for exactly solvable differential operators, J. Approx. Theory 149(2), (2007), 151-187.

[5] T. Bergkvist and H. Rullgård, On polynomial eigenfunctions for a class of differential operators, Math. Res. Lett., vol.9, (2002), 153-171.

[6] B. Shapiro, Algebro-geometric aspects of Heine-Stieltjes theory, arXiv:0812.4193.

[7] M. Fedoruyk, Asymptotic analysis. Linear ordinary differential equations, Translated from the Russian by Andrew Rodick. Springer-Verlag, Berlin, (1993), viii+363 pp.

[8] E. Heine, Handbuch der Kugelfunctionen, vol.1, Berlin: G. Reimer Verlag, (1878), 472-479.

[9] T. Holst, B. Kruglikov, B. Shapiro, Local study of multi-valued analytic differentials and their trajectories, in preparation.

[10] J. A. Jenkins, Univalent functions and conformal mapping. Ergebnisse der Mathematik und ihrer Grenzgebiete. Neue Folge, Heft 18. Reihe: Moderne Funktionentheorie Springer-Verlag, Berlin-Gttingen-Heidelberg, (1958), vi+169 pp.

[11] A. Martínez-Finkelshtein, E. A. Rakhmanov, Critical measures, quadratic differentials, and weak limits of zeros of Stieltjes polynomials, arXiv:0902.0193.

[12] A. Martínez-Finkelshtein and E. Saff, Asymptotic properties of Heine-Stieltjes and Van Vleck polynomials, J. Approx. Theory, vol. 118(1), (2002), 131-151.

[13] G. Pólya, Sur un théoreme de Stieltjes, C. R. Acad. Sci Paris vol. 155, (1912), 767-769.

[14] B. Shapiro, K. Takemura, M. Tater, On spectral polynomials of the Heun equation. II, in preparation.

[15] T. Stieltjes, Sur certains polynômes qui vérifient une équation différentielle linéaire du second ordre et sur la théorie des fonctions de Lamé, Acta Math. vol. 8, (1885), 321-326.

[16] K. Strebel, Quadratic differentials. Ergebnisse der Mathematik und ihrer Grenzgebiete (3) [Results in Mathematics and Related Areas (3)], 5. Springer-Verlag, Berlin, (1984), xii+184 pp.

[17] K. Strebel, On the metric $|f(z)|^{\lambda}|d z|$ with holomorphic $f$. Complex analysis (Warsaw, 1979), Banach Center Publ., 11, PWN, Warsaw, (1983), 323-336.

[18] G.Szegö, Orthogonal polynomials. Fourth edition. American Mathematical Society, Colloquium Publications, Vol. XXIII. American Mathematical Society, Providence, R.I., 1975. xiii+432 pp.

[19] E. Van Vleck, On the polynomials of Stieltjes, Bull. Amer. Math. Soc. vol. 4, (1898), 426-438.

[20] E. Whittaker and G. Watson, A course of modern analysis. An introduction to the general theory of infinite processes and of analytic functions; with an account of the principal transcendental functions. Reprint of the fourth (1927) edition. Cambridge Mathematical Library. Cambridge University Press, Cambridge, 1996. vi+608 pp.

Department of Mathematics, Stockholm University, SE-106 91 Stockholm, Sweden E-mail address: holst@math.su.se

Department of Mathematics, Stockholm University, SE-106 91 Stockholm, Sweden E-mail address: shapiro@math.su.se 